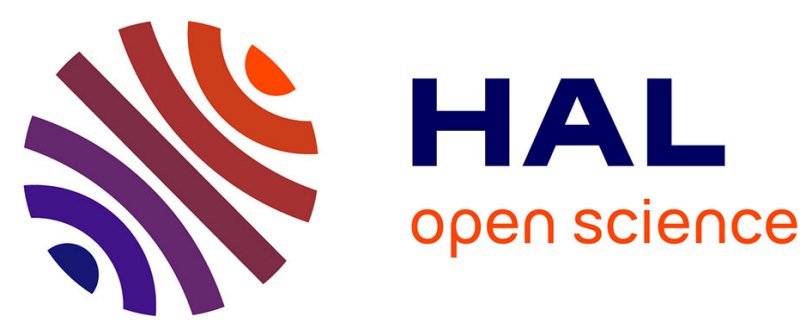

\title{
Multipoint concentration statistics for transport in stratified random velocity fields
}

Marco Dentz, Jesus Carrera, Diego Bolster, Tanguy Le Borgne

\section{To cite this version:}

Marco Dentz, Jesus Carrera, Diego Bolster, Tanguy Le Borgne. Multipoint concentration statistics for transport in stratified random velocity fields. Physical Review E : Statistical, Nonlinear, and Soft Matter Physics, 2009, 80, pp.036306. 10.1103/PhysRevE.80.036306 . insu-00562481

\section{HAL Id: insu-00562481 \\ https://hal-insu.archives-ouvertes.fr/insu-00562481}

Submitted on 3 Mar 2021

HAL is a multi-disciplinary open access archive for the deposit and dissemination of scientific research documents, whether they are published or not. The documents may come from teaching and research institutions in France or abroad, or from public or private research centers.
L'archive ouverte pluridisciplinaire HAL, est destinée au dépôt et à la diffusion de documents scientifiques de niveau recherche, publiés ou non, émanant des établissements d'enseignement et de recherche français ou étrangers, des laboratoires publics ou privés. 


\title{
Multipoint concentration statistics for transport in stratified random velocity fields
}

\author{
Marco Dentz ${ }^{*}$ and Jesus Carrera \\ Institute of Environmental Assessment and Water Research (IDAE-CSIC), 08034 Barcelona, Spain \\ Diogo Bolster \\ Department of Geotechnical Engineering and Geosciences, Technical University of Catalonia (UPC), 08034 Barcelona, Spain \\ Tanguy Le Borgne \\ Géosciences Rennes, UMR 6118, CNRS, Université de Rennes 1, 35042 Rennes, France \\ (Received 12 May 2009; revised manuscript received 17 July 2009; published 11 September 2009)
}

\begin{abstract}
We determine the full multipoint concentration statistics for superdiffusive transport in a steady stratified random velocity field. Using a Lagrangian approach, we derive explicit analytical expressions for the multipoint moments of concentration and specifically for the concentration variance, which is a measure for concentration uncertainty. The multipoint concentration moments are fully characterized by the Lagrangian mean velocity and by the one and two particle velocity correlations. While the relative variance at the center of mass of the mean concentration is constant, it increases exponentially with time and distance from the center of mass. This implies that small concentration values are particularly uncertain, which can pose a serious practical concern as these are typically the earliest and latest to arrive at a point.
\end{abstract}

DOI: 10.1103/PhysRevE.80.036306

PACS number(s): 47.60.-i, 92.40.Kf, 05.60.-k, 05.10.Gg

\section{INTRODUCTION}

Observed transport behavior in fluctuating environments is often anomalous. That is, the distribution of a transported quantity cannot be described by the classical advection dispersion equation (e.g., [1-4]). Even when transport may follow the advection-dispersion equation on a "local" scale, the effective large scale transport in natural environments does not. Spatiotemporal fluctuations can have a dual effect. On one hand, they act on the mean transport behavior and lead to effective transport dynamics that are different from their local scale counterpart. On the other hand, they introduce uncertainty due to limited knowledge on the fluctuation details. Oftentimes the only possible description of the fluctuating environment is statistical. Given such a statistical description, the transport behavior can be modeled by a stochastic approach. The latter provides a straightforward framework for both the quantification of the effective, mean transport behavior and the related uncertainty.

A series of applications require the quantification of both heterogeneity induced effective large scale transport and uncertainty. These include the safety assessment of geological hazardous waste facilities (e.g., $[5,6])$, groundwater management (e.g., $[7,8])$ and carbon sequestration in geological media (e.g., $[9,10])$. Another potential application is the movement of objects on the ocean surface and transport in turbulent and chaotic flows (e.g., [11]). Capturing the influence of heterogeneity can often be achieved by stochastic or spatial averaging over the local scale transport problem. In more general terms, one can perform a dimensional reduction or projection of the local scale processes. Such averaging implies a loss of information, which in general leads to a non-Markovianity property of the projected process (e.g.,

\footnotetext{
*marco.dentz@idaea.csic.es
}

[12]). Furthermore, spatial heterogeneity induces fluctuations about this mean behavior, which can be quantified by the ensemble variance of the distribution density $c(\mathbf{x}, t)$, which is defined by

$$
\sigma_{c}^{2}(\mathbf{x}, t)=\overline{[c(\mathbf{x}, t)-\bar{c}(\mathbf{x}, t)]^{2}},
$$

where the overbar denotes the ensemble average, $\bar{c}(\mathbf{x}, t)$ is the mean concentration. For transport in heterogeneous porous media the concentration variance has been studied in, e.g., Refs. [13-16] and is of general interest for transport in spatially fluctuating flow fields because it gives valuable information on the mixing properties of a passive scalar (e.g., $[11,17,18])$. In principle, the complete characterization of the process requires the knowledge of the full multipoint statistics of concentration. The latter have been looked at by using PDF (probability density functions)—-methods in, e.g., Refs. $[11,19,20]$. Frequently, the analysis of the concentration variance and the multipoint statistics is limited to perturbation theory and to so-called assumed PDF methods (e.g., [11,15]).

Here we present a nonperturbative, exact study of the multipoint concentration statistics for transport in a stratified random velocity field. The specific model under consideration has been introduced by [21] to study dispersion mechanism in heterogeneous porous media. Since then, this model has been subject to intense research (e.g., [3,22-25]) because it provides a relatively simple but nontrivial model to study the possible mechanisms that lead to superdiffusive behavior (i.e., a superlinear growth of the spatial variance) in heterogeneous media in particular and stratified random velocity fields in general. This model can be regarded as an approximation to aquifers characterized by vertical stratification often observed in geological media [26,27]. Figure 1 shows a realization of the type of velocity fields under consideration in this paper. Previously, [28] showed that effective transport in such a velocity field is non-Markovian and described by a Gaussian concentration distribution. Here we extend this 


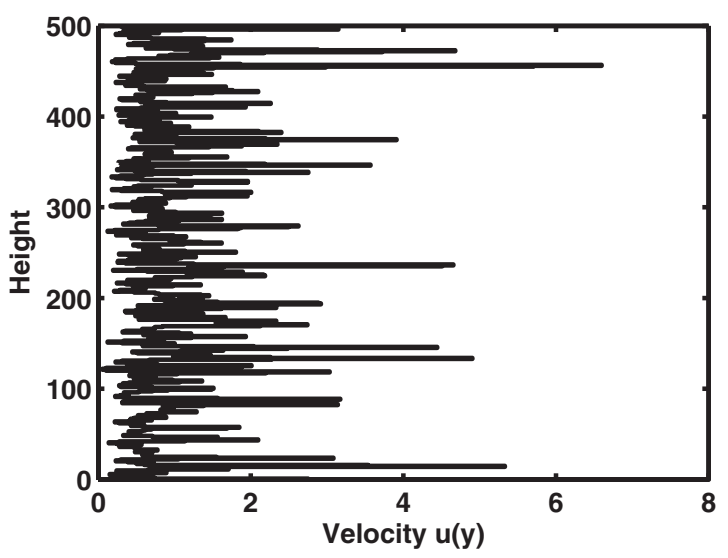
field.

FIG. 1. A sample realization of the considered random velocity

work and derive explicit expressions for the $n$-point moments of concentration.

\section{MODEL}

Transport of a passive scalar $g\left(\mathbf{x}, t \mid x_{2}^{\prime}\right)$ in a stratified flow field $u\left(x_{2}\right)$ can be described by the advection diffusion equation

$$
\frac{\partial g\left(\mathbf{x}, t \mid x_{2}^{\prime}\right)}{\partial t}=-u\left(x_{2}\right) \frac{\partial g\left(\mathbf{x}, t \mid x_{2}^{\prime}\right)}{\partial x_{1}}+D \frac{\partial^{2} g\left(\mathbf{x}, t \mid x_{2}^{\prime}\right)}{\partial x_{2}^{2}},
$$

where the coordinate vector is given by $\mathbf{x}=\left(x_{1}, x_{2}\right)^{T} ; D$ is the diffusion coefficient. For simplicity, we restrict this study to an infinite $d=2$ dimensional domain with no diffusion in the flow direction. The following analysis can be easily extended to more than two spatial dimensions and longitudinal diffusion. The spatial density $g\left(\mathbf{x}, t \mid x_{2}^{\prime}\right)$ and its gradients at infinity are zero. The initial distribution is given by $g(\mathbf{x}, t$ $\left.=t_{0} \mid x_{2}^{\prime}\right)=\delta\left(x_{1}\right) \delta\left(x_{2}-x_{2}^{\prime}\right)$ (which means that $g\left(\mathbf{x}, t=t_{0} \mid x_{2}^{\prime}\right)$ can be regarded as a Greens function). The stratified flow field $u\left(x_{2}\right)$ is a realization of a stationary spatial Gaussian random process $\left\{u\left(x_{2}\right)\right\}$ characterized by the constant ensemble mean velocity $u_{E}=E\left[u\left(x_{2}\right)\right]$ and correlation of the velocity fluctuations $u^{\prime}\left(x_{2}\right)=u\left(x_{2}\right)-u_{E}$, which is $C^{E}\left(x_{2}-x_{2}^{\prime}\right)$ $=E\left[u^{\prime}\left(x_{2}\right) u^{\prime}\left(x_{2}^{\prime}\right)\right]$. The notation $E[\cdot]$ stands for the ensemble average.

We consider now an ensemble of initial plumes that are distributed vertically according to $\rho\left(x_{2}\right) ; \rho\left(x_{2}\right)$ is normalized to one, its variance is denoted by $a^{2}$. For $a \gg 1$, it is assumed to have the scaling form

$$
\rho\left(x_{2}\right) \sim \frac{1}{a} f_{\rho}\left(\frac{x_{2}}{a}\right),
$$

where $f_{\rho}(0)$ is constant. Sampling the ensemble of concentration distributions along the vertical and taking the limit of $a \rightarrow \infty$ gives the mean concentration

$$
\bar{c}\left(x_{1}, t\right)=\lim _{a \rightarrow \infty} \frac{1}{a} \int_{-\infty}^{\infty} d x_{2}^{\prime} f_{\rho}\left(\frac{x_{2}^{\prime}}{a}\right) c\left(x_{1}, t \mid x_{2}^{\prime}\right),
$$

where we defined the projected concentration

$$
c\left(x_{1}, t \mid x_{2}^{\prime}\right) \equiv \int_{-\infty}^{\infty} d x_{2} g\left(\mathbf{x}, t \mid x_{2}^{\prime}\right) .
$$

The mean squared concentration is defined accordingly by

$$
\overline{c^{2}}\left(x_{1}, t\right)=\lim _{a \rightarrow \infty} \frac{1}{a} \int_{-\infty}^{\infty} d x_{2}^{\prime} f_{\rho}\left(\frac{x_{2}^{\prime}}{a}\right) c\left(x_{1}, t \mid x_{2}^{\prime}\right)^{2} .
$$

This averaging procedure is equivalent to performing the ensemble average over the concentration distribution evolving from a point injection because the random shear flow is assumed to be an ergodic random field (recall $a \gg 1$ ). In the following we derive explicit expressions for the multipoint moments of concentration in general and for the concentration variance $\sigma_{c}^{2}\left(x_{1}, t\right)=\overline{c^{2}}\left(x_{1}, t\right)-\bar{c}\left(x_{1}, t\right)^{2}$ in particular.

\section{ENSEMBLE STATISTICS}

Here we want to determine explicit equations for the evolution of the $n$-point statistics of the ensemble of projected concentration densities $\left\{c\left(x_{1}, t \mid x_{2}^{\prime}\right)\right\}$. The $n$-point density of $c\left(x_{1}, t \mid x_{2}^{\prime}\right)$ is defined by

$$
\phi_{n}(\mathbf{y}, t) \equiv \lim _{a \rightarrow \infty} \frac{1}{a} \int_{-\infty}^{\infty} d x_{2}^{\prime} f_{\rho}\left(\frac{x_{2}^{\prime}}{a}\right) \prod_{i=1}^{n} c\left(x_{1}^{(i)}, t \mid x_{2}^{\prime}\right) .
$$

where $\mathbf{y}=\left(x_{1}^{(1)}, \ldots, x_{1}^{(n)}\right)^{T}$. The simplest and most commonly used, statistical characteristics of $\left\{c\left(x_{1}, t \mid x_{2}^{\prime}\right)\right\}$ are its mean value (4), which is $\bar{c}\left(x_{1}, t\right)=\phi_{1}\left(x_{1}, t\right)$ and its variance (1). The latter is defined in terms of $\phi_{1}\left(x_{1}, t\right)$ and $\phi_{2}\left(x_{1}^{(1)}, x_{1}^{(2)}, t\right)$ as

$$
\sigma_{c}^{2}\left(x_{1}, t\right)=\phi_{2}\left(x_{1}, x_{1}, t\right)-\phi_{1}\left(x_{1}, t\right)^{2} .
$$

Let us begin by defining the "raw" $n$-point density

$$
\varphi_{n}\left(\mathbf{x}^{(1)}, \ldots, \mathbf{x}^{(n)}, t \mid x_{2}^{\prime}\right)=\prod_{i=1}^{n} g\left(\mathbf{x}^{(i)}, t \mid x_{2}^{\prime}\right),
$$

which satisfies the transport equation

$$
\begin{aligned}
\frac{\partial \varphi_{n}\left(\mathbf{x}^{(1)}, \ldots, \mathbf{x}^{(n)}, t \mid x_{2}^{\prime}\right)}{\partial t}= & -\sum_{i=1}^{n}\left[u\left(x_{2}^{(i)}\right) \frac{\partial}{\partial x_{1}^{(i)}}-D \frac{\partial^{2}}{\partial x_{2}^{(i) 2}}\right] \\
& \times \varphi_{n}\left(\mathbf{x}^{(1)}, \ldots, \mathbf{x}^{(n)}, t \mid x_{2}^{\prime}\right) .
\end{aligned}
$$

The initial condition is $\varphi_{n}\left(\mathbf{x}^{(1)}, \ldots, \mathbf{x}^{(n)}, t=t_{0} \mid x_{2}^{\prime}\right)$ $=\prod_{i=1}^{n} \delta\left(x_{1}^{(i)}\right) \delta\left(x_{2}^{(i)}-x_{2}^{\prime}\right)$. The $n$-point moment of concentration then is given by

$$
\begin{aligned}
\phi_{n}\left(\mathbf{y}, t \mid x_{2}^{\prime}\right)= & \lim _{a \rightarrow \infty} \frac{1}{a} \int_{-\infty}^{\infty} d x_{2}^{\prime} f_{\rho}\left(\frac{x_{2}^{\prime}}{a}\right) \\
& \times \int_{-\infty}^{\infty} d^{n} x_{2} \varphi\left(\mathbf{x}^{(1)}, \ldots, \mathbf{x}^{(n)}, t \mid x_{2}^{\prime}\right) .
\end{aligned}
$$

Equation (10) is a $2 n$-dimensional Fokker-Planck equation. It is exactly equivalent to the following system of Langevin equations (e.g., [29]), 


$$
\frac{d X_{1}^{(i)}\left(t \mid x_{2}^{\prime}\right)}{d t}=u_{E}+u^{\prime}\left[X_{2}^{(i)}\left(t \mid x_{2}^{\prime}\right)\right], \quad \frac{d X_{2}^{(i)}\left(t \mid x_{2}^{\prime}\right)}{d t}=\xi^{(i)}(t)
$$

for $i=1, \ldots, n$. The $\xi^{(i)}(t)$ denote Gaussian white noises with zero mean and correlation $\left\langle\xi^{(i)}(t) \xi^{(j)}\left(t^{\prime}\right)\right\rangle=\delta_{i j} 2 D \delta\left(t-t^{\prime}\right)$. The angular brackets denote the white noise average. The initial particle positions at time $t=t_{0}$ are given by $\left[X_{1}^{(i)}\left(t_{0}\right), X_{2}^{(i)}\left(t_{0}\right)\right]$ $=\left(0, x_{2}^{\prime}\right)$ for $i=1, \ldots, n$. The raw $n$-point density in this framework is given by

$$
\varphi\left(\mathbf{x}^{(1)}, \ldots, \mathbf{x}^{(n)}, t \mid \mathbf{x}^{\prime}\right)=\prod_{i=1}^{n}\left\langle\delta\left[\mathbf{x}^{(i)}-\mathbf{X}^{(i)}\left(t \mid x_{2}^{\prime}\right)\right]\right\rangle .
$$

Inserting Eq. (13) into Eq. (11), we obtain for the $n$-point density

$$
\phi_{n}\left(\mathbf{y}, t \mid x_{2}^{\prime}\right)=\int_{-\infty}^{\infty} d x_{2}^{\prime} \rho\left(x_{2}^{\prime}\right) \prod_{i=1}^{n}\left\langle\delta\left[x_{1}^{(i)}-X_{1}^{(i)}\left(t \mid x_{2}^{\prime}\right)\right]\right\rangle .
$$

\section{A. Averaging}

Now consider the $n$-dimensional system of Langevin equations,

$$
\frac{d Y_{i}(t)}{d t}=u_{E}+\zeta_{i}(t)
$$

for $i=1, \ldots, n$, where the initial positions are $Y_{i}\left(t=t_{0}\right)=0$ and the noise is defined by $\zeta_{i}(t)=u^{\prime}\left[X_{2}^{(i)}\left(t \mid x_{2}^{\prime}\right)\right]$. The noise has the same distribution as $u^{\prime}(y)$, which here is a Gaussian random field. Thus, $\zeta_{i}(t)$ is uniquely defined by its mean and variance

$$
\begin{aligned}
&\left\langle\zeta_{i}(t)\right\rangle_{\zeta}=\lim _{a \rightarrow \infty} \frac{1}{a} \int_{-\infty}^{\infty} d x_{2}^{\prime} f_{\rho}\left(\frac{x_{2}^{\prime}}{a}\right)\left\langle u^{\prime}\left[X_{2}^{(i)}\left(t \mid x_{2}^{\prime}\right)\right]\right\rangle, \\
&\left\langle\zeta_{i}(t) \zeta_{j}\left(t^{\prime}\right)\right\rangle_{\zeta}= \lim _{a \rightarrow \infty} \frac{1}{a} \int_{-\infty}^{\infty} d x_{2}^{\prime} f_{\rho}\left(\frac{x_{2}^{\prime}}{a}\right) \\
& \times\left\langle u^{\prime}\left[X_{2}^{(i)}\left(t \mid x_{2}^{\prime}\right)\right] u^{\prime}\left[X_{2}^{(j)}\left(t^{\prime} \mid x_{2}^{\prime}\right)\right]\right\rangle,
\end{aligned}
$$

where $\langle\cdot\rangle_{\zeta}$ denotes the noise average. Making use of the ergodicity of $u^{\prime}\left(x_{2}\right)$, we obtain that $\langle\zeta(t)\rangle_{\zeta}=0$ and (e.g., [28])

$$
\begin{gathered}
\left\langle\zeta_{i}(t) \zeta_{i}\left(t^{\prime}\right)\right\rangle_{\zeta}=\int_{-\infty}^{\infty} d x_{2} C^{E}\left(x_{2}\right) G\left(x_{2}, t-t^{\prime} \mid 0\right), \\
\left\langle\zeta_{i}(t) \zeta_{j}\left(t^{\prime}\right)\right\rangle_{\zeta}=\int_{-\infty}^{\infty} d x_{2} \int_{-\infty}^{\infty} d x_{2}^{\prime} C^{E}\left(x_{2}-x_{2}^{\prime}\right) G\left(x_{2}, t \mid 0\right) G\left(x_{2}^{\prime}, t^{\prime} \mid 0\right),
\end{gathered}
$$

where $i \neq j ; G\left(x_{2}, t \mid x_{2}^{\prime}\right)$ is the Green function for diffusion in vertical direction

$$
G\left(x_{2}, t \mid x_{2}^{\prime}\right)=\frac{1}{\sqrt{4 \pi D t}} \exp \left[-\frac{\left(x_{2}-x_{2}^{\prime}\right)^{2}}{4 D t}\right] .
$$

This is the marginal particle distribution in vertical direction. The $n$-point density (14) can now be written in terms of the $n$-dimensional correlated random walk (15) as

$$
\phi_{n}\left(\mathbf{y}, t \mid x_{2}^{\prime}\right)=\left\langle\prod_{i=1}^{n} \delta\left[x_{1}^{(i)}-Y_{i}(t)\right]\right\rangle_{\zeta} .
$$

\section{B. Explicit solutions for the $\boldsymbol{n}$-point density}

Discretizing Eq. (15) in time, we obtain

$$
\mathbf{Y}\left(t_{N}+\tau\right)=\mathbf{Y}\left(t_{N}\right)+\mathbf{u}_{E} \tau+\zeta\left(t_{N}\right) \tau
$$

with the constant time increment $\tau$ and discrete time $t_{N}=N \tau$, we defined the $n$-dimensional vector $\mathbf{u}_{E}=\left(u_{E}, \ldots, u_{E}\right)^{T}$. The random walk (22) has the sharp initial position $Y_{i}\left(t_{N_{0}}\right)=0$. The random force $\zeta\left(t_{N}\right)$ kicks in at time $t_{N_{0}}$. In order to make the process well defined, for $t_{N}<t_{N_{0}}$ we consider the process deterministic or driven by white noise. Thus, for $t_{N}>t_{N_{0}}$ the evolution of $\left.Y_{i}\left(t_{N}\right)\right]$ does not depend on the system states previous to $t_{N_{0}}$. The stochastic process given by the series of random velocities $\left\{\zeta_{i}\left(t_{l}\right)\right\}_{l=N_{0}}^{\infty}$ can be described by its characteristic function $\chi\left(\left\{\boldsymbol{\kappa}\left(t_{l}\right)\right\}\right)$ (e.g., [29]). For the multiGaussian correlated processes $\zeta(t)=\left[\zeta_{1}(t), \ldots, \zeta_{n}(t)\right]$, it is given by

$$
\chi\left(\left\{\boldsymbol{\kappa}\left(t_{l}\right)\right\}\right)=\exp \left[-\frac{1}{2} \sum_{l l^{\prime}} \sum_{i j=1}^{n} \kappa_{i}\left(t_{l}\right) C_{i j}^{L}\left(t_{l}, t_{l^{\prime}}\right) \kappa_{j}\left(t_{l^{\prime}}\right)\right],
$$

where from Eqs. (18) and (19) we define the Lagrangian velocity correlation $C_{i j}^{L}\left(t, t^{\prime}\right) \equiv\left\langle\zeta_{i}(t) \zeta_{j}\left(t^{\prime}\right)\right\rangle_{\zeta} ;\left\{\kappa_{i}\left(t_{n}\right)\right\}$ denote the Fourier variables conjugate to $\left\{\zeta_{i}\left(t_{n}\right)\right\}$. In order to derive an explicit expression for $\phi_{n}(\mathbf{y}, t)$ we perform a Fouriertransform of Eq. (21), which in discrete time gives

$$
\widetilde{\phi}_{n}\left(\mathbf{k}, t_{N}\right)=\left\langle\exp \left[i \mathbf{k} \cdot \mathbf{Y}\left(t_{n}\right)\right]\right\rangle_{\zeta},
$$

where $\mathbf{k}=\left(k_{1}, \ldots, k_{n}\right)^{T}$ is the $n$-dimensional wave vector. From Eq. (22) we obtain for the $n$-dimensional trajectory

$$
\mathbf{Y}\left(t_{N}\right)=\sum_{l=N_{0}}^{N-1} \mathbf{u}_{\mathbf{E}} \tau+\sum_{l=N_{0}}^{N-1} \zeta\left(t_{l}\right) \tau .
$$

Inserting this into Eq. (24), and performing the noise average gives

$$
\widetilde{\phi}_{n}\left(\mathbf{k}, t_{N}\right)=\exp \left[i \mathbf{k} \cdot \mathbf{u}_{E} \tau\right] \chi\left(\left\{\boldsymbol{\kappa}\left(t_{i}\right)=\mathbf{k}\right\}_{i=N_{0}}^{N-1} ;\left\{\boldsymbol{\kappa}\left(t_{i}\right)=0\right\}_{i \geq N}\right) .
$$

At large times, $t_{N} \gg \tau$, we take the limit to continuous time. Thus, from Eqs. (23) and (26)

$$
\widetilde{\phi}_{n}(\mathbf{k}, t)=\exp \left[-\mathbf{k} \cdot \int_{0}^{t-t_{0}} d t^{\prime} \mathbf{D}\left(t^{\prime}\right) \mathbf{k}+i \mathbf{k} \cdot \mathbf{u}_{E}\right],
$$

where we have defined the time dependent dispersion coefficients

$$
D_{i j}(t)=\int_{0}^{t} d t^{\prime} C_{i j}^{L}\left(t, t^{\prime}\right)
$$

Thus the $n$-point density in real space is given by 


$$
\phi_{n}(\mathbf{y}, t)=\frac{\exp \left[-\left(\mathbf{y}-\mathbf{u}_{E} t\right) \boldsymbol{\sigma}^{-2}\left(t-t_{0}\right) / 2\left(\mathbf{y}-\mathbf{u}_{E} t\right)\right]}{\sqrt{(2 \pi)^{n} \operatorname{det} \boldsymbol{\sigma}^{2}\left(t-t_{0}\right)}},
$$

where $\boldsymbol{\sigma}^{2}(t)$ is the tensor of trajectory variances

$$
\boldsymbol{\sigma}^{2}(t)=2 \int_{0}^{t} d t^{\prime} \mathbf{D}\left(t^{\prime}\right)
$$

and $\boldsymbol{\sigma}^{-2}(t)$ its inverse.

\section{Concentration variance}

The most commonly used measures of uncertainty are the mean and variance. The concentration variance has been frequently studied for transport in heterogeneous porous media (e.g., $[13-15,30,31])$ in both Eulerian and Lagrangian modeling frameworks. Oftentimes, these studies are limited to perturbation theory in the velocity fluctuations. We focus here on the concentration mean and variance as indicators of uncertainty and the self-averaging properties of concentration (e.g., [1]). From Eq. (29), we obtain for the concentration variance $(8)$ the compact expression

$$
\begin{aligned}
\sigma_{c}^{2}\left(x_{1}, t\right)= & \bar{c}\left(x_{1}, t\right)^{2} \\
& \times\left(\frac{\exp \left\{\left[x_{1}-u_{E}\left(t-t_{0}\right)\right]^{2} \frac{\sigma_{12}^{2}}{\sigma_{11}^{4}+\sigma_{12}^{2} \sigma_{11}^{2}}\right\}}{\sqrt{1-\sigma_{12}^{4} / \sigma_{11}^{4}}}-1\right),
\end{aligned}
$$

where the mean concentration is given by [28]

$$
\bar{c}\left(x_{1}, t\right)=\frac{\exp \left\{-\frac{\left[x_{1}-u_{E}\left(t-t_{0}\right)\right]^{2}}{2 \sigma_{11}^{2}}\right\}}{\sqrt{2 \pi \sigma_{11}^{2}}} .
$$

In the case of a delta-correlation in the 2-direction, that is $C^{E}\left(x_{2}\right)=\sigma_{u}^{2} l \delta\left(x_{2}\right)$ with $\sigma_{u}^{2}$ the variance of the velocity field and $l$ its correlation length, the dispersion coefficients are (e.g., $[21,32,33])$

$$
D_{i i}(t)=\frac{\sigma_{u}^{2} \tau_{D}}{\sqrt{\pi}} \sqrt{t / \tau_{D}}, \quad D_{i j}(t)=(\sqrt{2}-1) D_{i i}(t)
$$

We define the diffusion time scale $\tau_{D}=l^{2} / D$. Inserting Eq. (33) into Eq. (31), we obtain for the relative variance

$$
\frac{\sigma_{c}^{2}\left(x_{1}, t\right)}{\bar{c}\left(x_{1}, t\right)^{2}}=\frac{\exp \left\{\left[x_{1}-u_{E}\left(t-t_{0}\right)\right]^{2} \frac{\sqrt{2}-1}{2 \sqrt{2} \sigma_{11}^{2}}\right\}}{\sqrt{2 \sqrt{2}-2}}-1 .
$$

At the center of mass, the relative concentration variance is constant and given by $\frac{\sigma_{c}^{2}\left[u_{E}\left(t-t_{0}\right), t\right]}{c\left[u_{E}\left(t-t_{0}\right), t\right]^{2}} \approx 0.1$. The concentration variance decreases exponentially with time and distance from the center of mass of the mean concentration. The relative variance, in contrast, increases exponentially with time at positions that do not coincide with the center of mass of the mean distribution, because $\sigma_{11}^{2} \propto\left(t-t_{0}\right)^{3 / 2}$ in Eq. (34). Furthermore, the relative variance increases exponentially
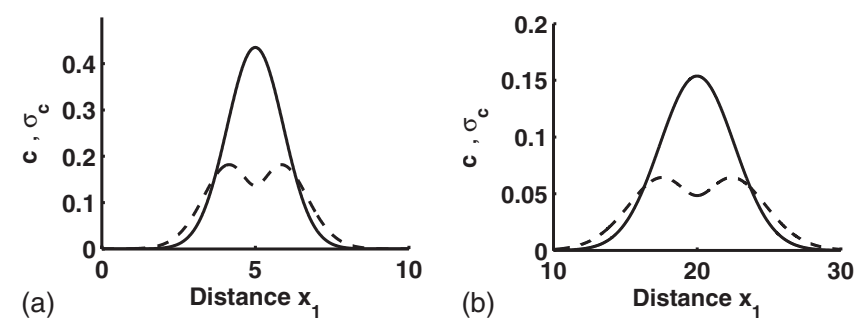

FIG. 2. Evolution of the mean concentration (solid) and variance (dashed) at two times (a) $t=500 \tau_{D}$ and (b) $t=2 \times 10^{3} \tau_{D}$ for a variance of $\sigma_{u}^{2}=1$.

with distance from the mean center of mass, which implies that small concentrations are particularly uncertain. In particular, these observations imply that the concentration is not self-averaging.

From the outset such a behavior is not completely unexpected because the dispersion behavior in infinitely extended stratified media has been found to be non-self-averaging either. This is indicated by the fact that the variance of the center of mass fluctuations of the plume, as quantified by the two-particle variance $\sigma_{12}^{2}(t)$, increases superdiffusively with time. The latter marks the difference between two dispersion measures which are called the ensemble and effective dispersion coefficients (e.g., [32,34]). While for transport in nonstratified divergence-free random velocity fields, the two dispersion measures converge in the asymptotic long time limit, for stratified media they are always different [32]. In fact, the relative variance as expressed by Eq. (31) would tend to zero if the two-particle variance $\sigma_{12}(t)^{2}$ tended to zero. For a confined stratified medium this may be the case.

Figure 2 shows the mean concentration $\bar{c}\left(x_{1}, t\right)$ and standard deviation $\sigma_{c}\left(x_{1}, t\right)$ at $t=500 \tau_{D}$ and $t=2 \times 10^{3} \tau_{D}$. Note the double peak in the standard deviation, which indicates that the region of maximum uncertainty and maximum average concentration do not coincide. The general shapes of the curves change proportionally in time (reflecting the fact that the ratio between $D_{11}(t)$ and $D_{12}(t)$ is constant).

\section{Numerical simulations}

The explicit analytical expression (31) for the concentration variance is compared to Monte Carlo simulations. We use particle tracking simulations in two-dimensional random shear flow. The simulated flow field is organized in layers of constant thickness $l$. The velocity within each layer is constant, drawn from a Gaussian distribution with unit mean and variance. For transverse scales much larger than the thickness of a layer, the correlation is approximately delta (e.g., [33]). Solute transport is simulated by random walk particle tracking. Initial positions are uniformly distributed along a vertical line source, whose length is $10^{4} l$. Within each stratum $10^{3}$ particles are released, which gives a total of $10^{7}$ simulated particle trajectories. Averages are then taken over the projected partial distributions that evolve from the strata. The implementation of the particle tracking method is described in detail in [35]. Equivalently, we considered averages over particle distributions consisting of $10^{3}$ particles evolving from point sources in $10^{4}$ different realizations of 


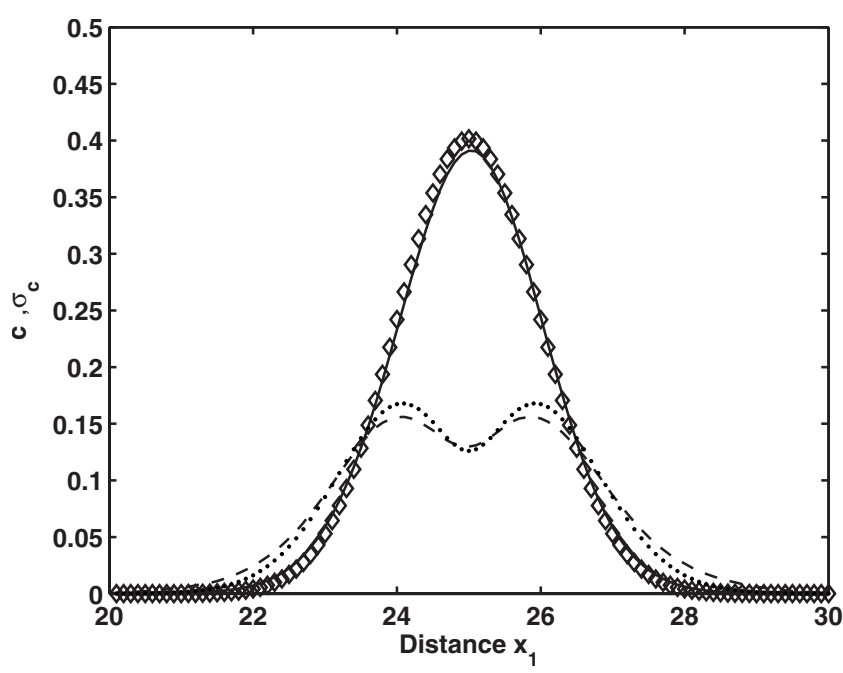

FIG. 3. A comparison between the numerical concentration (-) and standard deviation (- -) against the analytical values of concentration $(\diamond)$ and standard deviation $(\cdot)$ for $\sigma_{u}^{2}=1$ and $t=2.5$ $\times 10^{5} \tau_{D}$

the flow field. The results are identical as a consequence of the ergodicity of the random velocity field. In the following, we present only the results obtained for a long line source in a single realization.

A comparison of the results from the numerical simulations and the corresponding analytical solutions are shown in Fig. 3. As one can see, the analytical and numerical solutions are in good agreement, which is not surprising as the derived expressions for the multipoint concentration moments are exact for an infinite medium. Several other simulations varying the modeling parameters were run, yielding similar results. Note that the mean concentration obtained by numerical simulations agrees almost perfectly with the predicted behavior, while for the variance there are slight deviations. This can be traced back to the fact that the latter is much more sensitive to noise.

\section{SUMMARY AND CONCLUSIONS}

We present a Lagrangian methodology for deriving the multipoint concentration statistics in Gaussian distributed random shear flow and determine explicit expressions for the multipoint concentration moments. The specific model under consideration has been frequently considered in the literature to study non-Fickian diffusion and the resulting effective particle densities (e.g., $[3,21,22,24,25,28])$. In fact, the effective dispersion coefficient evolves anomalously with the square root of time. In a stochastic modeling approach, the mean concentration distribution of a solute is defined by ensemble averaging of the concentration distribution originating from an instantaneous point injection in one realization of the random flow field.

Alternatively, for an ergodic random flow field, the mean behavior can be obtained by considering transport in a single realization for a solute that originates from a line source perpendicular to the stratification that is much larger than the characteristic scale of flow stratification. In this case the mean behavior is obtained by projecting the partial plumes evolving from points along the vertical line source and averaging of the source distribution [28]. The vertical particle movements describe a Brownian motion and are independent of the longitudinal movement. Thus the effective distribution is given by the product of the vertically projected effective density and a Gaussian density representing the vertical distribution. Therefore, it is sufficient to focus on the vertical projection of the effective particle distribution. Dentz et al. [28] showed that the effective, or mean, particle movements can be represented by a correlated random walk, where the noise correlation is uniquely defined by the Lagrangian velocity correlation function. The mean particle distribution is then given by the density of this random walk. Being a correlated random walk, the process is non-Markovian. Here we extend this methodology to determine the full concentration statistics as given by the explicit analytical expressions for the multipoint moments of the concentration distribution. This yields a full statistical characterization of the nonMarkovian effective particle dynamics. We obtain multiGaussian distribution densities for the $n$-point densities. These are completely characterized by (i) the mean particle velocity, (ii) the single particle velocity correlation, and (iii) the two-particle velocity correlation.

We then study the concentration variance as a simple measure for the uncertainty of concentration values and for the self-averaging properties of the particle distribution. The concentration variance is characterized by a double peak and decreases with time and distance from the center of mass. The relative concentration, in contrast, increases both with distance from the mean center of mass and time. This implies that the low concentration values are particularly uncertain, or in other words, that the low probabilities of finding a particle at a given position at a given time, are very uncertain. The relative variance is constant at the center of mass of the mean distribution which indicates that the probability of the bulk of the particles, or the highest concentration, is subject to a basically constant uncertainty.

These results have some practical relevance because experimentally determined concentration values and particle densities are often obtained as space and/or time averages and the observed mean behavior is often found to be superdiffusive. The presented work considers a model that gives some insight in the mechanism that can lead to such behavior and provides a methodology to quantify uncertainty and in fact the full concentration statistics.

In conclusion, by focusing on a relatively simple random flow model, this work sheds some new light and provides novel insights on the features and possible limitations of stochastic transport models in random flows. The presented methodology and the results obtained may have some impact for risk assessment studies and extreme value analysis. 
[1] J. P. Bouchaud and A. Georges, Phys. Rep. 195, 127 (1990).

[2] B. Berkowitz, A. Cortis, M. Dentz, and H. Scher, Rev. Geophys. 44, RG2003 (2006).

[3] W. R. Young and S. Jones, Phys. Fluids A 3, 1087 (1991).

[4] E. Frey and K. Kroy, Ann. Phys. 14, 20 (2005).

[5] J. Carrera, J. Contam. Hydrol. 13, 23 (1993).

[6] B. Berkowitz and H. Scher, Transp. Porous Media 42, 241 (2001).

[7] D. Tartakovsky, Geophys. Res. Lett. 34, L05404 (2007).

[8] D. Bolster, M. Barahona, M. Dentz, D. Fernandez-Garcia, X. Sanchez-Vila, P. Trinchero, C. Valhondo, and D. M. Tartakovsky, Water Resour. Res. 45, W06413 (2009).

[9] J. A. Neufeld and H. E. Huppert, J. Fluid Mech. 625, 353 (2009).

[10] D. Bolster, M. Dentz, and J. Carrera, Water Resour. Res. 45, W05408 (2009).

[11] S. Pope, Turbulent Flows (Cambridge University Press, Cambridge, England, 2000).

[12] R. Kubo, M. Toda, and N. Hashitsume, Statistical Physics II, Non-Equilibrium Statistical Mechanics (Springer-Verlag, Berlin, Heidelberg, 1991).

[13] V. Kapoor and L. W. Gelhar, Water Resour. Res. 30, 1775 (1994).

[14] V. Kapoor and P. K. Kitanidis, Water Resour. Res. 34, 1181 (1998).

[15] V. Kapoor and P. K. Kitanidis, Stoch. Hydrol. Hydraul. 11, 397 (1997).

[16] W. Graham and D. McLaughlin, Water Resour. Res. 25, 215 (1989).

[17] S. Fedotov, M. Ihme, and H. Pitsch, Phys. Rev. E 71, 016310 (2005).
[18] Z. Warhaft, Annu. Rev. Fluid Mech. 32, 203 (2000).

[19] E. Caroni and V. Fiorotto, Transp. Porous Media 59, 19 (2005).

[20] O. A. Cirpka, R. L. Schwede, J. Luo, and M. Dentz, J. Contam. Hydrol. 98, 61 (2008).

[21] G. Matheron and G. de Marsily, Water Resour. Res. 16, 901 (1980).

[22] J. P. Bouchaud, A. Georges, J. Koplik, A. Provata, and S. Redner, Phys. Rev. Lett. 64, 2503 (1990).

[23] S. Redner, Physica A 168, 551 (1990).

[24] G. Zumofen, J. Klafter, and A. Blumen, Phys. Rev. A 42, 4601 (1990).

[25] S. N. Majumdar, Phys. Rev. E 68, 050101(R) (2003).

[26] O. Güven, F. Molz, and J. G. Melville, Water Resour. Res. 20, 1337 (1984).

[27] A. Fiori and G. Dagan, Transp. Porous Media 47, 81 (2002).

[28] M. Dentz, T. LeBorgne, and J. Carrera, Phys. Rev. E 77, 020101(R) (2008).

[29] H. Risken, The Fokker-Planck Equation (Springer, Heidelberg, New York, 1996).

[30] A. Fiori and G. Dagan, J. Contam. Hydrol. 45, 139 (2000).

[31] E. Morales-Casique, S. P. Neuman, and A. Guadagnini, Adv. Water Resour. 29, 1238 (2005).

[32] M. Clincy and H. Kinzelbach, J. Phys. A 34, 7141 (2001).

[33] V. Zavala-Sánchez, M. Dentz, and X. Sanchez-Vila, Adv. Water Resour. 32, 635 (2008)

[34] M. Dentz, H. Kinzelbach, S. Attinger, and W. Kinzelbach, Water Resour. Res. 36, 3591 (2000).

[35] T. Le Borgne, J. R. De Dreuzy, P. Davy, and O. Bour, Water Resour. Res. 43, W02419 (2007). 